\title{
Several Remarks on Grover's Quantum Search Algorithm with a Single Marked Element

\author{
Qi Han ${ }^{1}$, Yanan $\mathrm{Han}^{1 *}$, Ziqiang $\mathrm{Lu}^{1}$, Yaxin $\mathrm{Kou}^{1}$
}

${ }^{1}$ College of Mathematics and Statistics, Northwest Normal University, Lanzhou, 730070, Gansu P.R. China

DOI: $10.36347 /$ sipms.2021.v08i03.002

| Received: 09.02.2021 | Accepted: 19.03.2021 | Published: 28.03.2021

*Corresponding author: Yanan Han

Abstract

Review Article

The Grover's quantum search algorithm is one of the most important quantum algorithms. In this article, we mainly discuss related properties of the Grover's quantum search algorithm with a single marked element. We show that the range of constant $c$ when the probability of measured value $x_{0}$ is $p$.

Keywords: Grover's algorithm; unitary operator; reflection operators; optimality.

Copyright $\left({ }_{0} 2021\right.$ The Author(s): This is an open-access article distributed under the terms of the Creative Commons Attribution 4.0 International License (CC BY-NC 4.0) which permits unrestricted use, distribution, and reproduction in any medium for non-commercial use provided the original author and source are credited.

\section{INTRODUCTION}

Search algorithm is a common algorithm. The efficiency of classical computer to the data search is relatively low, and the computational complexity is $O(N)$, where $N$ is the number of database entries. Correspondingly, the Grover's quantum search algorithm in quantum computers, and the time complexity of Grover's algorithm is $O(\sqrt{N})$. Grover's quantum search algorithm originally designed to look for an element in an unsorted database with no repeated elements. In the case of a large number of database entries, the superiority of Grover's quantum search algorithm is extremely obvious. Grover's algorithm can realize the quadratic acceleration of classical algorithm; therefore, Grover's quantum search algorithm is introduced on this basis which has a wide applicability $[1,2,3,9]$.

The evolution operator and the initial condition of Grover's algorithm have real entries, then means that the entire evolution takes place in a real vector subspace of the Hilbert space $\mathrm{H}^{2 N}$ [1]. However, the key to this algorithm is that evolution operator $U$ is the product of two reflection operators $R_{x_{0}{ }^{\perp}}$ and $R_{D}$, where $R_{x_{0}{ }^{\perp}}$ is a reflection operator around the vector space spanned by the vector orthogonal to $\left|x_{0}\right\rangle$ with point $x_{0}$ is called a marked element, $R_{D}$ is a reflection operator around the vector space spanned by $|D\rangle$. In real vector spaces, the operation of two successive reflection operators on the initial vector will cause the initial vector to rotate by an angle. Starting from initial condition $|D\rangle$, one application of $U$ rotates $|D\rangle$ approximately by $\frac{2}{\sqrt{N}}$ degrees toward $\left|x_{0}\right\rangle$. So far, no algorithm can find the marked element faster than Grover's algorithm and the probability of success is greater than or equal to $1-\frac{1}{N}$. There are many other interesting results about Grover's quantum search algorithm. For example, the Grover's algorithm searches for repeated elements and amplitude amplification technique and operator coherence dynamics of Grover's algorithm $[6,7,8,10,11]$.

In this paper, we show that the related properties of Grover's quantum search algorithm with single marked element. In Section 2, we briefly describe some concepts of Grover's algorithm. In Section 3, we show that related properties of unitary evolution and optimality of Grover's algorithm. In Section 4, we have a briefly summarize of the whole article.

\section{Some concepts of Grover's algorithm}

Definition 2.1 [5] (Classical search algorithm): Suppose that $f$ is a function with domain 
Qi Han et al., Sch J Phys Math Stat, Mar, 2021; 8(3): 62-67

$\{0, \cdots, N-1\}$, where $N=2^{n}$ and $n$ is some positive integers, and image

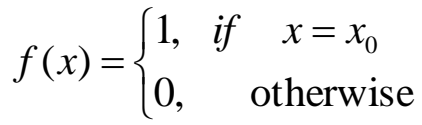

The function image is 1 only for a single point $x_{0}$ and 0 for all other points. In this problem, exhaustive search on point $x_{0}$ is carried out through query function $f$, and the time complexity of classical algorithm is $\Omega(N)$, function $f$ is called query database, and point $x_{0}$ is called marked element.

In $[1,4]$, for the quantum context, the unitary operator is used based on function $f$, and the full description of this operator is called $R_{f}$, in the computational basis is

$$
R_{f}|x\rangle|i\rangle=|x\rangle|i \oplus f(x)\rangle
$$

Here $\oplus$ means addition in modulo 2 .

Grover's algorithm uses a second unitary defined by

$$
R_{D}=\left(2|D\rangle\langle D|-I_{N}\right) \otimes I_{2},
$$

Where $|D\rangle$ the diagonal state of the first is register, $I_{N}$ and $I_{2}$ are unit operators of $N$ dimensional and 2 dimensional space, respectively. Thus the evolution operator that performs one step of the algorithm is

$$
U=R_{D} R_{f} \text {. }
$$

The initial condition is $\left|\psi_{0}\right\rangle=|D\rangle|-\rangle$ Where $|-\rangle=\frac{|0\rangle-|1\rangle}{\sqrt{2}}$.

Remark 2.1 [1] (The process of Grover's quantum search algorithm) When the Grover's algorithm input is $N$ and $f$ in formula (2.1), and when the following four conditions are fulfilled

1, Use a 2 -register quantum computer with $n+1$ quantum bits;

2, Prepare the initial state $|D\rangle|-\rangle$;

3, Apply $U^{t}$, where $t=\left\lfloor\frac{\pi}{4} \sqrt{N}\right\rfloor$ and $U$ is given by

4, Measure the first register in the computational basis, Where \lfloor\rfloor is the integral to the left of a real number?
Then the output of the algorithm is $x_{0}$ and its probability is greater than or equal to $1-\frac{1}{N}$.

\section{Basic properties of unitary evolution and optimality of Grover's algorithm}

In $[1,5,6]$, the evolution operator and the initial condition of Grover's algorithm have real entries, this means that the entire evolution takes place in a real vector subspace of the Hilbert space $\mathrm{H}^{2 N}$, the key of the algorithm is the product operator $U$ of two reflection operators $R_{f}$ and $R_{D}$. Let $\left|x_{0}^{\perp}\right\rangle$ be a unit vector that is orthogonal to $\left|x_{0}\right\rangle$, which is in the plane spanned by $\left|x_{0}\right\rangle$ and $|D\rangle$ and has the smallest angle with $|D\rangle$, the expression for $\left|x_{0}^{\perp}\right\rangle$ in the computer is $\left|x_{0}^{\perp}\right\rangle=\frac{1}{N-1} \sum_{x \neq x_{0}}|x\rangle$, and in the evolution of the plane spanned by $\left|x_{0}\right\rangle$ and

$$
\begin{gathered}
|D\rangle \text {, the operator } R_{f} \text { can be replaced by } R_{x_{0}{ }^{\perp}}, \\
R_{x_{0} \perp}=2\left|x_{0}^{\perp}\right\rangle\left\langle x_{0}^{\perp}\right|-I_{N} .
\end{gathered}
$$

Therefore, the evolution operator $U$ of the Grover's algorithm is replaced by $U=R_{D} R_{x_{0}^{\perp}}$, where the initial condition is $|D\rangle$.

The initial condition of Grover's algorithm is state $|D\rangle$. After applying the operator $R_{f}$, state $|D\rangle$ is reflected around the plane orthogonal to vector $\left|x_{0}\right\rangle$. After applying operator $R_{D}$, vector $R_{f}|D\rangle$ is reflected around $|D\rangle$. That is, one application of $U$ rotates the initial vector by $\theta$ degrees toward vector $\left|x_{0}\right\rangle$.

Lemma 3.1 [1] Let $\frac{\theta}{2}$ be the angle between vectors $\left|x_{0}^{\perp}\right\rangle$ and $|D\rangle$, which is the complement of the angle between $\left|x_{0}^{\perp}\right\rangle$ and $|D\rangle$. So

$$
\sin \frac{\theta}{2}=\cos \left(\frac{\pi}{2}-\frac{\theta}{2}\right)=\left\langle x_{0} \mid D\right\rangle=\frac{1}{\sqrt{N}},
$$

When function $f$ has a large domain, we obtain

$$
\theta=\frac{2}{\sqrt{N}}+\frac{1}{3 N \sqrt{N}}+O\left(\frac{1}{N^{2}}\right)
$$


Qi Han et al., Sch J Phys Math Stat, Mar, 2021; 8(3): 62-67

and at $t_{f}=\left\lfloor\frac{\pi}{4} \sqrt{N}\right\rfloor$, the probability of finding $x_{0}$ is

$$
p_{x_{0}}=\left|\left\langle x_{0}\left|U^{t_{f}}\right| D\right\rangle\right|^{2}=\cos ^{2} \frac{\theta}{2}=1-\frac{1}{N} \text {. }
$$

After understanding basic concepts of the algorithm, we discuss some fundamental properties of the algorithm. For example, properties of unitary operator and the rotation angle $\theta$.

lemma 3.1 For a diagonal state $|D\rangle$, the $t$-th power of evolution operator $U$ has the following form:
$U^{t}|D\rangle=\sin \left(t \theta+\frac{\theta}{2}\right)\left|x_{0}\right\rangle+\operatorname{sos}\left(t \theta+\frac{\theta}{2}\right)\left|x_{0}^{\perp}\right\rangle$.

In order to understand the relationship between the probability of success (find the marked element) and the probability of failure (unfound the marked element), we give the following proposition.

Proposition 3.2 For all case $x \neq x_{0}$ and $x=x_{0}$, the sum of the probability of Grover's algorithm at $N \geq 1$ is asymptotically equal to 1 .

Proof. The known fact:

$$
\begin{gathered}
P_{x_{0}}=\left|\left\langle x_{0}\left|U^{t_{f}}\right| D\right\rangle\right|^{2}, \\
U^{t_{f}}|D\rangle=\sin \left(t_{f} \theta+\frac{\theta}{2}\right)\left|x_{0}\right\rangle+\cos \left(t_{f} \theta+\frac{\theta}{2}\right)|x\rangle, \\
\theta=\frac{2}{\sqrt{N}}+\frac{1}{3 N \sqrt{N}}+O\left(\frac{1}{N^{2}}\right), t_{f}=\left|\frac{\pi}{4} \sqrt{N}\right|, \\
P_{x}=\left|\left\langle x\left|U^{t_{f}}\right| D\right\rangle\right|^{2}=\left|\cos \left(t_{f} \theta+\frac{\theta}{2}\right)\right|^{2}=\cos ^{2}\left(t_{f} \theta+\frac{\theta}{2}\right) \\
=\left[\cos \left(\frac{\pi}{4} \sqrt{N} \times \frac{2}{\sqrt{N}}+\frac{1}{2} \frac{2}{\sqrt{N}}\right)\right]^{2} \\
=\left[\cos \left(\frac{\pi}{2}+\frac{1}{\sqrt{N}}\right)\right]^{2}=\sin ^{2} \frac{1}{\sqrt{N}} \approx\left|\frac{1}{\sqrt{N}}\right|^{2}=\frac{1}{N}
\end{gathered}
$$

and

$$
P_{x_{0}}=\sin ^{2}\left(\frac{\pi}{2}+\frac{1}{\sqrt{N}}\right)=\cos ^{2} \frac{1}{N}=\frac{1-\cos \frac{2}{\sqrt{N}}}{2} \approx \frac{1+1-\frac{2}{N}}{2}=1-\frac{1}{N} \text {. }
$$

So

$$
P_{x}+P_{x_{0}} \approx 1
$$

In $[1,5,6,7]$, the Grover's quantum search algorithm finds the marked element by querying the oracle $O(\sqrt{N})$ times. And have proved that Grover's algorithm is optimal, that is, no algorithm can find the marked element faster than Grover's algorithm and the probability of success is greater than or equal to $1-\frac{1}{N}$, by [1] we can know that the $\left|\psi_{0}\right\rangle$ be the initial state, the quantum state after $t$ steps is given by

$$
\left|\psi_{t}\right\rangle=U_{t} R_{f} \cdots U_{1} R_{f} U_{0}\left|\psi_{0}\right\rangle
$$

Where $U_{1}, \cdots, U_{t}$ are generic unitary operators, further, the quantum state is defined as

$$
\left|\phi_{t}\right\rangle=U_{t} \cdots U_{0}\left|\psi_{0}\right\rangle \text {, }
$$

by iteration of general unitary operators. 
To prove the optimality of the Grover's algorithm, one way that is to compare state $\left|\psi_{t}\right\rangle$ and $\left|\phi_{t}\right\rangle$, then define a quantity again

$$
\left.D_{t}=\frac{1}{N} \sum_{x_{0}=0}^{N-1} \| \psi_{t}\right\rangle-\left|\phi_{t}\right\rangle \|^{2}
$$

Which measures the deviation between $\left|\psi_{t}\right\rangle$ and $\left|\phi_{t}\right\rangle$ after $t$ steps.

Through [1, 7], the Grover's quantum search algorithm is optimal when the following inequality is true

$$
c \leq D_{t} \leq \frac{4 t^{2}}{N}
$$

Where $c$ is a strictly positive constant.

We know that the constant $c$ is related to the probability of measuring the return value of $x_{0}$, without loss of generality, we will talk about the range of constant $c$ when the probability is a general number $p$.

Proposition 3.3 If the probability of measurement value $x_{0}$ is greater than or equal to $p$, the constant $c$ is in the range of

$$
0<c<(\sqrt{2}-\sqrt{2-2 \sqrt{p}})^{2} .
$$

Proof. According to the optimality of Grover's algorithm, the following formula holds:

$$
c \leq D_{t} \leq \frac{4 t^{2}}{N}
$$

Where $D_{t}=\frac{1}{N} \sum_{x_{0}=0}^{N-1} \|\left|\psi_{t}\right\rangle-\left|\phi_{t}\right\rangle \|^{2},\left|\psi_{t}\right\rangle=U_{t} R_{f} \cdots U_{1} R_{f} U_{0}\left|\psi_{0}\right\rangle,\left|\phi_{t}\right\rangle=U_{t} \cdots U_{0}\left|\psi_{0}\right\rangle$,

$\left|\psi_{0}\right\rangle$ is the initial state, $R_{f}=I-2\left|x_{0}\right\rangle\left\langle x_{0}\right|, U_{1}, \cdots, U_{t}$ are generic unitary operators.

We know the fact that $D_{t} \leq \frac{4 t^{2}}{N}$, so let us prove the range of $c$. Let us define two new quantities given by

$$
\left.E_{t}=\frac{1}{N} \sum_{x_{0}=0}^{N-1} \|\left|\psi_{t}\right\rangle-\left|x_{0}\right\rangle\left\|^{2}, F_{t}=\frac{1}{N} \sum_{x_{0}=0}^{N-1}\right\| \phi_{t}\right\rangle-\left|x_{0}\right\rangle \|^{2} .
$$

We obtain an inequality involving $D_{t}, E_{t}$ and $F_{t}$ as following:

$$
\begin{aligned}
& D_{t}=\frac{1}{N} \sum_{x_{0}=0}^{N-1} \|\left|\psi_{t}\right\rangle-\left|\phi_{t}\right\rangle \|^{2} \\
& =\frac{1}{N} \sum_{x_{0}=0}^{N-1} \|\left(\left|\psi_{t}\right\rangle-\left|x_{0}\right\rangle\right)+\left(\left|x_{0}\right\rangle-\left|\phi_{t}\right\rangle\right) \|^{2} \\
& \geq \frac{1}{N} \sum_{x_{0}=0}^{N-1}\left[\|\left|\psi_{t}\right\rangle-\left|x_{0}\right\rangle\left\|^{2}-2\right\|\left|\psi_{t}\right\rangle-\left|x_{0}\right\rangle\|\|\left|\| \phi_{t}\right\rangle-\left|x_{0}\right\rangle\|+\|\left|x_{0}\right\rangle-\left|\phi_{t}\right\rangle \|^{2}\right] \\
& =E_{t}+F_{t}-\frac{2}{N} \sum_{x_{0}=0}^{N-1} \|\left|\psi_{t}\right\rangle-\left|x_{0}\right\rangle|\||\left|\phi_{t}\right\rangle-\left|x_{0}\right\rangle \| \\
& \left.\geq E_{t}+F_{t}-\frac{2}{N} \sum_{x_{0}=0}^{N-1}\left(\| \psi_{t}\right\rangle-\left|x_{0}\right\rangle \|\left.\right|^{\frac{1}{2}}\left(\| \phi_{t}\right\rangle-\left|x_{0}\right\rangle \|\right)^{\frac{1}{2}} \\
& =E_{t}+F_{t}-2 \sqrt{E_{t} F_{t}}=\left(\sqrt{F_{t}}-\sqrt{E_{t}}\right)^{2} .
\end{aligned}
$$


We now show that $F_{t} \geq 2-2 \frac{1}{\sqrt{N}}$, define $\theta_{x_{0}}$ as the phase of $\left\langle x_{0} \mid \phi_{t}\right\rangle$, that is

$$
\left\langle x_{0} \mid \phi_{t}\right\rangle=e^{i \theta_{x_{0}}}\left|\left\langle x_{0} \mid \phi_{t}\right\rangle\right| \text {. }
$$

Define the state

$$
\begin{gathered}
|\theta\rangle=\frac{1}{\sqrt{N}} \sum_{x_{0}=0}^{N-1} e^{i \theta_{x_{0}}}\left|x_{0}\right\rangle . \\
\left\langle\theta \mid \phi_{t}\right\rangle=\frac{1}{\sqrt{N}} \sum_{x_{0}=0}^{N-1} e^{-i \theta_{x_{0}}}\left\langle x_{0} \mid \phi_{t}\right\rangle \\
=\frac{1}{\sqrt{N}} \sum_{x_{0}=0}^{N-1}\left|\left\langle x_{0} \mid \phi_{t}\right\rangle\right|=\frac{1}{\sqrt{N}} \times N=\sqrt{N} .
\end{gathered}
$$

Using the Cauchy-Schwarz inequality, we obtain $\left|\left\langle\theta \mid \phi_{t}\right\rangle\right| \leq 1$, we use the above inequality and the fact that the real part of $\left\langle x_{0} \mid \phi_{t}\right\rangle$ is smaller than or equal to $\left|\left\langle x_{0} \mid \phi_{t}\right\rangle\right|$ :

$$
\begin{aligned}
& F_{t}=\frac{1}{N} \sum_{x_{0}=0}^{N-1} \|\left|\phi_{t}\right\rangle-\left|x_{0}\right\rangle \|^{2} \\
& =\frac{1}{N} \sum_{x_{0}=0}^{N-1}\left(\left\langle\phi_{t}\left|-\left\langle x_{0}\right|\right)\left(\left|\phi_{t}\right\rangle-\left|x_{0}\right\rangle\right)\right.\right. \\
& =\frac{1}{N} \sum_{x_{0}=0}^{N-1}\left(\left\langle\phi_{t} \mid \phi_{t}\right\rangle-\left\langle\phi_{t} \mid x_{0}\right\rangle-\left\langle x_{0} \mid \phi_{t}\right\rangle+\left\langle x_{0} \mid x_{0}\right\rangle\right) \\
& =2-\frac{2}{N} \operatorname{Re}\left\{\left\langle x_{0} \mid \phi_{t}\right\rangle\right\} \geq 2-\frac{2}{N} \sum_{x_{0}=0}^{N-1}\left|\left\langle x_{0} \mid \phi_{t}\right\rangle\right| \geq 2-\frac{2}{\sqrt{N}}
\end{aligned}
$$

After $t$ steps, the state of the quantum computer after the application of the oracles is $\left|\psi_{t}\right\rangle$. Similar to the calculation used for $F_{t}$, we have

$$
E_{t}=\frac{1}{N} \sum_{x_{0}=0}^{N-1} \|\left|\psi_{t}\right\rangle-\left|x_{0}\right\rangle \|^{2}=2-\frac{2}{N} \sum_{x_{0}=0}^{N-1} \operatorname{Re}\left\{\left\langle x_{0} \mid \psi_{t}\right\rangle\right\} \text {. }
$$

Let us assume that the probability of a measurement to return value $x_{0}$ is greater than or equal to $p$, that is, $\left|\left\langle x_{0} \mid \psi_{t}\right\rangle\right|^{2} \geq p$ for all $x_{0}$. Value $p$ is arbitrary between 0 and 1 . we use basis $\left\{e^{i \alpha_{0}}|0\rangle, \cdots, e^{i \alpha_{N-1}}|N-1\rangle\right\}$, where $\alpha_{x_{0}}$ for $0 \leq x_{0} \leq N$ is define as the phase of $\left\langle x_{0} \mid \psi_{t}\right\rangle$. In this basis, $\left\langle\tilde{x}_{0} \mid \psi_{t}\right\rangle$ is a real number, that is, $\operatorname{Re}\left\{\left\langle\tilde{x}_{0} \mid \psi_{t}\right\rangle\right\}=\left|\left\langle\tilde{x}_{0} \mid \psi_{t}\right\rangle\right|$.

Therefore,

$$
\begin{aligned}
& E_{t}=2-\frac{2}{N} \sum_{x_{0}=0}^{N-1} \operatorname{Re}\left\{\left\langle\tilde{x}_{0} \mid \psi_{t}\right\rangle\right\} \\
& =2-\frac{2}{N} \sum_{x_{0}=0}^{N-1}\left|\left\langle\tilde{x}_{0} \mid \psi_{t}\right\rangle\right| \leq 2-\frac{2}{N} \sum_{x_{0}=0}^{N-1} \sqrt{p} \\
& =2-\frac{2}{N} \times N \sqrt{p}=2-2 \sqrt{p}
\end{aligned}
$$

Using inequalities $E_{t} \leq 2-2 \sqrt{p}$ and $F_{t}=\geq 2-\frac{2}{\sqrt{N}}$, We obtain

$$
\begin{aligned}
& D_{t} \geq\left(\sqrt{F_{t}}-\sqrt{E_{t}}\right)^{2} \geq\left(\sqrt{2-\frac{2}{\sqrt{N}}}-\sqrt{2-2 \sqrt{p}}\right)^{2} \\
& =(\sqrt{2}-\sqrt{2-2 \sqrt{p}})^{2}+O\left(\frac{1}{\sqrt{N}}\right) .
\end{aligned}
$$

This completes the proof of inequality $c \leq D_{t}$ for $N$ large enough. Constant $c$ must obey

$$
0<c<(\sqrt{2}-\sqrt{2-2 \sqrt{p}})^{2} .
$$

\section{CONCLUSIONS}

In this article, we prove the related properties of unitary evolution in Grover's quantum search algorithm, and prove the range of constant $c$ when the probability of the measured value is $p$. However, we can further discuss properties related to the Grover's quantum search algorithm ability to find elements in a database of repeating elements and its algorithm optimality.

\section{REFERENCES}

1. Portugal R. Quantum walks and search algorithms. New York: Springer; 2013 Feb 16.

2. Grover LK. Quantum mechanics helps in searching for a needle in a haystack. Physical review letters. 1997 Jul 14;79(2):325.

3. Grover LK. Quantum computers can search rapidly by using almost any transformation. Physical Review Letters. 1998 May 11;80(19):4329.

4. Aharonov D. Quantum computation. Annual Reviews of Computational Physics VI. 1999:259346.

5. Bennett $\mathrm{CH}$, Bernstein E, Brassard G, Vazirani U. Strengths and weaknesses of quantum computing. SIAM journal on Computing. 1997 Oct;26(5):1510-23.

6. Nielsen MA and Chuang I. Quantum computation and quantum information; 2002.

7. Zalka C. Grover's quantum searching algorithm is optimal. Physical Review A. 1999 Oct $1 ; 60(4): 2746$.

8. Taha MM, Perkowski M. Realization of arithmetic operators based on stochastic number frequency signal representation. In2018 IEEE 48th 
Qi Han et al., Sch J Phys Math Stat, Mar, 2021; 8(3): 62-67

International Symposium on Multiple-Valued Logic (ISMVL) 2018 May 16 (pp. 215-220). IEEE.

9. Zhang W. Quantum Algorithms for Two-Arm robot and generalization to Travelling Salesman Problem (2020, in Preparation); 2020.

10. Pan M, Qiu D. Operator coherence dynamics in Grover's quantum search algorithm. Physical Review A. 2019 Jul 31;100(1):012349.

11. Liu L, Xiao M, Song X. Authenticated semiquantum dialogue with secure delegated quantum computation over a collective noise channel. Quantum Information Processing. 2018 Dec;17(12):1-7. 